\title{
Esthétique culinaire dans vi de Kim Thúy
}

Kyeongmi Kim-Bernard, Université MacEwan

L'écriture migrante, dont le balbutiement est surtout perceptible ici et ailleurs sur le globe depuis les années 1980, est suivie de façon croissante depuis le développement des moyens de transport et d'échange entre les nations. Elle arrive aujourd'hui à un statut étonnant étant donné l'explosion en nombre de ses productions et de ses résonnances littéraires. Un écrivain migrant est celui qui s'est éloigné de son pays natal, que ce soit de façon forcée ou volontaire, et qui se trouve sur un sol d'accueil au moment de sa création littéraire. Janet Patterson résume les raisons du départ d'un écrivain migrant en quatre aspects différents en citant l'analyse de Edward Saïd : les exilés, les réfugiés, les expatriés et les émigrés (89). Si les vingt premières années ont vu plus d'écrivains exilés et réfugiés, depuis le début du nouveau siècle, les expatriés et les émigrés, eux, se manifestent de plus en plus. Cela se traduit également avec un changement de tendance dans l'écriture migrante. Si nous avons vu plus de récits de l'exil, de la perte et de la dépossession, résultant en l'écriture d'une identité difficile à construire dans un espace entre-deux, qui se montrait en un enracinement difficile chez les personnages de la première période, la nouvelle tendance va vers une poétique de l'écriture migrante transnationale qui, selon Paterson, se caractérise par des stratégies discursives favorisant la transcendance des «critères identitaires de la nation et de l'ethnie pour promouvoir des identités multiples, mouvantes, souvent multi-culturelles » (89).

Notamment au Canada, les écrivains tels que Ying Chen, Ook Chung, Dany Laferrière et Kim Thúy se démarquent de par leur écriture, qui fait preuve de leur penchant pour une nouvelle forme de quête faisant éclater les normes identitaires hétérogènes. Si les deux premiers écrivains sont issus des voies de l'expatriation et de l'émigration, les deux derniers sont plutôt marqués par leurs parcours d'exilé et de réfugié. Peu importe leur arrivée sur le sol d'accueil par des moyens forcés ou volontaires, dans leur écriture, la quête identitaire récuse la notion d'une identité formée sur le modèle traditionnel encadré par la couleur d'une ethnie ou bien par le topo d'une frontière géographique. La nouvelle identification est plutôt fondée sur la transgression de ces valeurs traditionnelles et atteint une identité «complexe et mouvante souvent multi-culturelle»(89). Dans cette étude, je me propose d'explorer cette nouvelle esthétique de l'écriture migrante, notamment à l'aide des images culinaires qui forment une stratégie discursive sensorielle importante dans l'écriture migrante transnationale de l'écrivaine montréalaise d'origine vietnamienne, Kim Thúy. 
«Dis-moi ce que tu manges, je te dirai qui tu es » : ce dicton français nous semble être une devise que Thúy a en tête lorsqu'elle écrit ses fictions et non-fictions. Afin de comprendre la vie migrante qui tisse les axes principaux de ses fictions, le lecteur est invité à suivre les parcours de ses protagonistes subtilement métaphorisés par les images culinaires découlant de leurs parcours migratoires. Thúy a dû endurer un double déracinement de son identité culturelle, causée par la colonisation de son pays natal par la France pendant un siècle, jusqu'en 1954, ensuite par l'immigration de sa famille au Québec, marquée par un exil forcé talonnant le sort des boat people à l'âge de dix ans. Ainsi, son écriture, indéniablement marquée par un transfert interculturel et international, une « écriture de l'entre-deux » selon Régine Robin (69), parvient à une ultime tentation de fusion culturelle en brouillant les frontières. Les parcours migratoires dans les écrits de Thúy se veulent une trajectoire qui passe par une construction identitaire transnationale et pluriculturelle.

Dans son plus récent roman vi (2016), après $R u$ (2009), À toi (2011) et Mãn (2013), Thúy use efficacement des images culinaires afin d'évoquer les aspects saillants de la quête identitaire de la narratrice éponyme qui se déroule dans plusieurs villes : Québec, Montréal, Saigon, Hanoï, etc. Ici, il serait pertinent de mentionner le parcours professionnel atypique que l'écrivaine a eu elle-même avant d'arriver à l'écriture ; Thúy a d'abord été avocate, ensuite restauratrice, avant de publier son premier roman $R u$ en 2009. Comme l'indique son intérêt pour la cuisine qui lui a permis de travailler professionnellement pendant cinq ans en ouvrant un restaurant vietnamien nommé « Ru de Nam » à Montréal (Dusaillant-Fernandes, « Habiller le vécu », 164), l'écrivaine réserve un rapport exceptionnel avec la matière culinaire. Par ailleurs, dans $v i$, les affirmations identitaires de la vie «nomadique » de la narratrice s'expriment efficacement à l'aide d'images culinaires de différentes provenances qui s'affrontent et qui s'apparient. Que ce soit le Vietnam natal qu'elle a dû quitter en bas âge comme boat people, tout comme l'écrivaine elle-même, puis le Québec, la terre d'accueil qui lui permet d'incorporer ses divers héritages culturels, et le Vietnam communiste où elle choisit de retourner travailler avec le statut ambigu d'une «étrangère », sans oublier l'empreinte douloureuse mais incontournable de la France sur la base gustative vietnamienne, les images culinaires sont présentes, mettant un éclairage efficace au dédale migratoire qu'est la vie de la narratrice. Par ailleurs, l'auteure a confié, dans un entretien accordé à la journaliste australienne Brigid Delaney le 19 mai 2015, que la valeur hautement symbolique, surtout au Vietnam, de l'acte de préparer et de partager la nourriture a formé une partie importante de sa personne et de son imaginaire : «Vietnamese don't usually verbalise their emotions - good or bad. They 
express their love through food. As soon as you step through the door of someone's home, they will ask you if you have eaten... ».

Dans ce contexte, il m'apparait pertinent d'analyser ce roman autofictionnel vi autour des papilles gustatives vietnamiennes ainsi que leurs rencontres avec d'autres saveurs alimentaires que le personnage principal du roman Vi goûte et incorpore dans ses registres sensoriels, voire identitaires. À travers cette étude je tenterai donc de démontrer comment les images culinaires peuvent devenir des moyens efficaces quant à la disposition culturelle et identitaire d'un personnage romanesque.

\section{Images culinaires comme une mise en conscience de la vietnamité}

Dans le roman vi, le Vietnam est présenté autour de deux périodes distinctes : le Vietnam d'avant le départ de la narratrice âgée de dix ans comme boat people et le Vietnam de son retour comme avocate internationale et « étrangère » dans son pays d'origine. La présence du Vietnam, la terre natale de Vi, représente surtout un là-bas à la fois traumatique et idéalisé que la mère de la narratrice tente à tout prix de préserver dans l'identité migrante de ses trois fils et de sa fille Vi. La mère de Vi, qui a vécu une double déchirure suite à l'émigration (l'exode du pays natal et l'abandon de son mari), s'accroche démesurément à la représentation identitaire du pays d'origine. Son attachement effréné à la coutume et à l'alimentation vietnamiennes qu'elle insiste à partager avec ses quatre enfants, évoque l'image du souvenir passionnel traumatisant du Vietnam et du mari dont elle a été désunie par force. Cette mère use de toute son influence pour préserver chez ses enfants le goût et l'odorat de leur pays d'origine comme s'ils combleraient le manque de la paternité et de la patrie pour cette famille éclatée et déportée. En effet, le dévouement de cette mère pour une transmission culturelle à ses enfants dans le roman $R u$ est considéré comme un élément principal de l'écriture migrante de Thúy dans l'analyse du Care qu'a effectué Marie Carrière dans son article «Mémoire du Care, féminisme en mémoire », mais cet aspect devient un élément à surmonter dans le roman $v i$. Vi se rappelle l'appartement de sa famille immigrée au Québec où planait constamment le souhait de la mère incorporé dans les parfums des aliments qu'elle préparait :

En faisant abstraction des bottes dans l'entrée et des manteaux d'hiver qui s'empilaient sur les lits, nous aurions pu prétendre être de retour à Saigon. Le parfum typique des cuisines vietnamiennes embaumait l'air grâce à ma mère. Elle nous plongeait dans l'odeur de la citronnelle hachée et rôtie mariée à la peau croustillante des poissons, ou dans celle des jeunes pousses de bambou sautées puis trempées dans la sauce de poisson à la lime. (54-55) 
Bien entendu, le choix exclusif des goûts et de l'odorat vietnamiens que la mère veut incorporer au quotidien de ses enfants a pour but de compenser l'absence de la patrie et du père qu'ils ont brusquement perdus lors du départ. Par ailleurs, Pamela V. Sing va jusqu'à appeler ce pouvoir gustatif que la mère de la narratrice déploie afin de perpétuer la vietnamité une «pratique translocalisante » (286). Bien entendu, ce terme évoque une tentative « translocalisante » de la part de la mère, traitement qui s'avère efficace à l'aide du goût pour cette famille démembrée. Grâce à l'odorat qui plane dans l'habitat et au goût des aliments vietnamiens, l'espace perdu du pays natal est symboliquement renouvelé dans l'imaginaire de la famille de Vi.

Par ailleurs, les souvenirs olfactifs évoque à la fois la nostalgie et le trauma de la déchirure que la narratrice garde de son pays natal. Dans la mémoire de Vi, la sauce de poisson formant la base de la cuisine maternelle sur le sol d'exil, est notamment un signal sensoriel qui lui évoque les souvenirs légers et heureux qu'elle garde de son père dans son pays natal :

Pendant toute mon enfance, nous allions à la mer presque tous les mois pour « changer de vents », comme disait mon père... L'air salin faisait grandir mes frères et amplifiait nos rires autour des seiches séchées vendues sur la plage par des marchands ambulants. Deux seiches complètement aplaties et grillées sur quelques charbons rouges nourrissaient la famille entière pendant tout l'aprèsmidi puisqu'elles se mangeaient fibre par fibre. En bouche, le goût de ces filaments élastiques durait plus longtemps qu'une gomme Juicy Fruit. (64)

La seiche, l'ingrédient principal de la sauce de poisson que la mère utilise, est le poisson dont le goût et l'odeur sont marqués par une intensité gustative et une insistance olfactive prolongées et capables d'interpeller seuls les vrais adeptes. Pour la mère de Vi, ce poisson devient l'élément par excellence d'une particularité culinaire vietnamienne. Dans ce contexte, le père qui a abandonné sa femme et ses enfants, et la patrie qui les a poussés à fuir, deviennent deux absences à combler chez ses enfants par le moyen de sa cuisine généreusement arrosée de cette sauce symbolique. De cette pratique culinaire, le petit appartement du Québec, ainsi rempli de l'odeur et de la saveur de cette sauce, forme une bulle exceptionnelle et «translocalisante » où la mère de la narratrice tente d'alimenter la tradition familiale et nationale malgré les deux déchirures traumatisantes qui les hantent. Par ailleurs, lors de l'interview déjà mentionnée avec Delaney, qui lui a demandé quels sont «les ingrédients indispensables » dans la cuisine vietnamienne, Thúy confie la valeur symbolique que cette sauce de poisson a pour les Vietnamiens : «We eat a lot of raw vegetables. There are not many dishes which are served without some mixture of greens - banana flowers, lotus stems, morning glory, green papaya... mixed with all kinds of herbs. But the must-have ingredient - as 
Marguerite Duras has listed as her essentials - is nuoc mam, the fish sauce. Without fish sauce, I think my dad would not survive more than 48 hours ».

La pratique culinaire est également l'élément principal qui permet à la famille d'appartenir à la diaspora vietnamienne encadrant l'identité de la narratrice dans son enfance. Les familles vietnamiennes exilées qui l'entourent, survivent ensemble le déracinement en mijotant des plats traditionnels, notamment lors des fêtes, telles que le Nouvel An traditionnel, le Têt :

Ainsi, le matin de ces dimanches précédant le Têt, ma mère, Hoa et moi nous réveillions très tôt pour préparer les herbes, les fines tranches de porc, les crevettes coupées en deux sur la longueur avant de les envelopper dans les galettes de riz. Même si nous étions trois personnes aux mains différentes, il fallait que tous les rouleaux aient la même taille, incluant les trois centimètres de ciboulette aillée qui en dépassaient fièrement comme des antennes. Les jeunes achetaient nos rouleaux, les raviolis farcis, les pâtés chauds, le gâteau au manioc, alors que les mères se promenaient entre les kiosques pour zieuter les jeunes filles célibataires qu'une amie ou connaissance leur avait recommandées pour leurs garçons. (80- 81)

L'identité vietnamienne, confirmée et échangée avec les autres immigrés, apparaît dans le choix des plats dont les ingrédients et la préparation s'acheminent exclusivement vers une vietnamité acharnée. Cet aspect de la mère de Vi voulant s'accrocher à la tradition culinaire du pays natal marque incontestablement la caractéristique d'un « roman de transmission et d'héritage » pour emprunter le terme à Valérie Dusaillant-Fernandes («Du Vietnam au Québec », 75). Le premier roman de Thúy, $R u$, est notamment marqué par cette caractéristique qui met l'accent sur le déracinement brusque et le difficile enracinement sur la terre d'accueil malgré la «phase euphorique » de l'arrivée sur le sol d'exil, mais dans $v i$, le récit tente de la dépasser en montrant une évolution marquée par plus d'ouverture vers d'autres cultures en passant par une revalorisation identitaire chez la narratrice. Cette tradition culinaire du pays d'origine maintenue par la mère ne peut, donc, pas éviter une mise à l'épreuve au fur et à mesure de la formation identitaire pluriculturelle que Vi et ses frères sont forcés de vivre dans la société de l'exil.

\section{La nourriture, moyen de séduction et de maintien conjugal}

Si la tradition culinaire, ainsi préservée et pratiquée par la mère, a pour mission de perpétuer la vietnamité qui fait défaut dans le milieu de la diaspora, la pratique culinaire s'avère également être une arme redoutable pour la séduction de l'homme aimé pour la mère de la narratrice. Le père de $\mathrm{Vi}$, à la fois adoré et haï dans son souvenir, tout comme la patrie, est 
décrit comme un beau garçon d'une famille influente de Saigon. En dépit du déséquilibre entre deux jeunes, que ce soit le physique ou bien la classe sociale qui les distinguent, la mère de Vi a réussi à l'épouser grâce aux soins culinaires extraordinaires dont le jeune homme ne pouvait plus se passer. La description de leur première rencontre ensorcelée par l'odorat et le goût exceptionnels d'un café que la jeune hôtelière d'une région montagneuse reculée qu'était la mère de $\mathrm{Vi}$, a préparé pour son futur mari, est particulièrement pertinente :

Étonné du goût distinct et soyeux du café, il a tourné les yeux vers ma mère. Elle lui en a confié le secret en lui montrant une petite boule difforme sertie de grains, ramassée dans les environs des plantations à Buôn Mê Thuôt. Ces boules provenaient des civettes sauvages qui rejetaient les grains tout entiers après avoir mangé et digéré les cerises de café mûres...Mon père est devenu instantanément adepte. Ma mère s'est portée volontaire pour être son fournisseur et celle qui lui détaillerait les arômes ajoutés avec parcimonie pendant la torréfaction, dont le précieux beurre importé de France. (21)

Cette séduction, par le culinaire et l'olfactif, devient le garant d'une vie conjugale fondée sur un déséquilibre physique et social. L'affection de la jeune femme envers l'homme aimé se montre surtout dans la description des plats qu'elle prépare avec dévouement pour lui :

Selon mon père, même les boulangers parisiens ne pouvaient se mesurer à ma mère. D'ailleurs, même s'il mangeait dans les meilleurs restaurants de la ville, il nous répétait qu'aucun chef ne savait retirer les fleurs de courgette farcies de la poêle comme elle, juste à temps pour préserver la texture des pétales. Seule ma mère en maîtrisait la cuisson et parvenait à faire ressortir leur sucre sous le croustillant de la légère panure à la farine de riz. Comme les autres familles vietnamiennes, nous présentions tous nos plats au milieu de la table en même temps, à une exception près. Ma mère servait mon père dans des assiettes séparées des nôtres afin de lui réserver le meilleur : le crabe à carapace moelle le plus gorgé d'œufs, les bâtonnets de pommes de terre frites parfaitement ordonnés, les feuilles de chicorée les plus tendres... Il allait de soi que la cinquantaine de pépins de la pomme cannelle étaient enlevés et sa chair blanche et mielleuse lui était tendue comme une offrande. (40-41)

La mère de $\mathrm{Vi}$, qui incarne la vieille tradition austère vietnamienne, est une femme dévouée à son mari, tout comme à la patrie dont l'espace, interrompu par un départ brutal, est renouvelé de façon constante par sa pratique culinaire quotidienne sur le sol d'adoption. Pour elle, la nourriture qu'elle prépare pour l'être aimé, est la seule véritable expression de son sentiment qui n'est pas censé être verbalisée selon la tradition vietnamienne, contrairement à celle, occidentale, qui « encourage l'expression des sentiments et des opinions (59) », constat prononcé par sa fille Vi. Cette magie culinaire, qui lie le mari à la femme, se perpétue dans la génération suivante chez le frère de $\mathrm{Vi}$, marié et homme d'affaires à succès à Montréal. La mère de Vi prépare des plats destinés à son fils Long, qu'elle passe à sa bru Hoa lorsqu'il faut rapprocher le couple éloigné : «Elle observait le retour à la maison de Long. S'il arrivait trop 
tard trop souvent, elle lui préparait ses plats préférés. Elle l'appelait au travail sans insister, sans mentionner qu'une famille l'attendait, sans lui rappeler de résister au désir. Elle livrait tout simplement les plats à Hoa et espérait entendre un rire ou deux à travers les murs » (123).

Pour la mère de Vi, cette pratique culinaire s'avère un moyen d'extérioriser son existence à part entière. Que ce soit son affection conjugale et maternelle ou bien celle d'une vietnamité à préserver chez ses enfants, l'apport gustatif s'avère le véhicule efficace pour transmettre les messages de cette femme. La perception culinaire ainsi léguée à Vi par sa mère connaître, pourtant, une déroute qui l'invitera à un élargissement gustatif à cause de son contact avec d'autres propriétés sensorielles au fur et à mesure de l'évolution identitaire de la jeune femme.

\section{Déterritorialisation culinaire}

$\mathrm{Vi}$, qui retourne au Vietnam devenu un pays communiste vingt ans après son brusque départ, découvre un aspect important de son pays natal ignoré jusqu'alors dans la mémoire des exilés de la diaspora vietnamienne. Hanoï, la capitale située au nord du Vietnam, est doublement étrangère pour la jeune avocate canadienne d'origine de Saigon. Cette fois-ci encore, une certaine altérité de ce pays d'origine est observée par le moyen culinaire dans le roman :

J'ai tenté de rattraper quelques bribes des vingt ans du Vietnam derrière le rideau de fer en trainant autour des tables-restaurants. Devant mon hôtel, il y en avait plusieurs. L'une offrait des baguettes au pâté de foie, l'autre, des vermicelles sautés, et plusieurs, des soupes tonkinoises. Je finissais mes journées avec cette soupe qui ne ressemblait en rien à celle cuisinée à Montréal, Los Angeles, Paris, Sydney ou Saigon. La version hanoïenne se vendait seulement avec quelques tranches de bœuf saignant alors que j'avais toujours mangé ce plat avec une dizaine d'ingrédients, dont les tendons, l'estomac, le jarret, du basilic thaï, des fèves germées... (102)

Le fait d'être une Occidentale d'adoption sur le territoire d'Orient de son origine, met la narratrice Vi dans une interrogation de conscience identitaire particulière. Le pays natal idéalisé dans la mémoire de la diaspora en Occident se concrétise dans la préparation de la soupe Phô consommée avec de multiples ingrédients aromatiques et fantaisistes par les immigrants dans leurs villes d'exil, tandis que la nouvelle identité récusée par ces exilés se présente tout autrement devant Vi dans l'évocation de la soupe Phô tonkinoise cuisinée sans faste qu'elle mange dans la capitale du pays communiste. Cette altérité absorbée et digérée au sein de son pays natal marque bien entendu un tournant primordial quant au rapport de la narratrice Vi avec sa mère et son pays natal. Pour la jeune narratrice, « trop occidentale » selon 
le critère de sa mère, la barrière qui la limitait dans une identité tracée et prédestinée par sa mère et par la diaspora vietnamienne, éclate ici tout comme la nouvelle réalité de sa patrie qu'elle constate tous les jours à Hanoi. Dès lors, cette mise en conscience de l'altérité au sein du soi, qui s'avère avoir composé son identité à son insu, devient un signal de son besoin d'ouverture vers d'autres mondes et d'autres apports gustatifs. Par ailleurs, dans une interview accordée à Jean-François Plamondon, l'auteur a livré une confidence quant au climat interculturel dans lequel elle était forcée de grandir avant de quitter le Vietnam. La colonisation du Vietnam par la France a, néanmoins, permis d'avoir, malgré des effets dévastateurs, un aspect positif sur le plan culturel :

...mais en réalité, la littérature française a influencé la littérature vietnamienne et à mon avis, c'est un des plus importants apports de la colonisation française en Indochine. Il y a eu au Vietnam une osmose entre les imaginaires occidentales et asiatiques. Les Français de l'Indochine n'ont d'ailleurs pas moins été marqués par notre culture que nous par la leur... Cela se répercute même dans les traditions culinaires. Pour la majorité des Vietnamiens, le flan caramel est un dessert vietnamien... (158-159)

L'osmose culturelle, décrite par une influence réciproque pendant la colonisation française au Vietnam, ne peut pas se passer sans une interpénétration entre les deux cultures colonisatrice et colonisée en dépit de la domination d'une partie sur l'autre. Cette interpénétrabilité de deux cultures joue le rôle du médium pour d'autres ouvertures à l'altérité, chez la narratrice Vi.

Comme l'aspect saillant de l'écriture migrante au Québec constaté par Simon Harel dans son livre Braconnages identitaires, d'abord les constants éloges de «l'éclatement» identitaire avant d'arriver à une envie d'une « re-territorialisation » identitaire, deux traits qui sont répétés comme un processus sans fin, Vi passe par cet éclatement de son identité primaire avant d'enlacer un nouveau territoire identitaire. Suivant ce processus d'éclatement, une créolisation culinaire s'impose sur la table. Ce terme de créolisation, découlant de la langue créole, est pris dans le sens qu'Édouard Glissant a voulu lui donner comme l'objectif de la pratique de l'écriture migrante. Dans «L'anthropophagie dans les littératures des Amériques », Jessica Gaudette résume la créolisation glissantienne comme un «processus perpétuel dont la dynamique résulte des rencontres entre diverses singularités. L'homme, conscient d'être traversé par le divers qui compose la totalité du monde est ainsi libéré de toute prétention à l'universel. Cette prise de conscience dévoile également l'importance qu'a l'impact de sa propre participation à la diversité du tout-monde » (191-192). Cette perspective de créolisation est aussi pertinente dans l'écriture migrante de Thúy, notamment dans ce roman $v i$. 
Ainsi, la palette culinaire vietnamienne se marie avec de multiples autres sources d'aliments étrangers et enrichit le plaisir gustatif de la narratrice Vi. Le changement culinaire que celle-ci expérimente, se passe en même temps que sa rencontre à Hanoi avec Vincent, un écologiste-ornithologue d'origine française. Le retour de Vi au Vietnam natal, décidé suite à sa rupture d'avec Tân, son premier amour et immigrant comme elle au Québec, devient paradoxalement un moment décisif de l'ouverture vers d'autres mondes comme, par exemple, la cuisine française, trace imposante d'une colonisation dans la pratique culinaire de la mère, qui est revalorisée chez la narratrice depuis sa rencontre avec Vincent. Cette redéfinition de la valeur gustative française donne lieu à un certain rapprochement de deux habitudes culinaires à sa table. Notamment, Vi prépare des plats qui reflètent son ouverture vers d'autres aliments et leur communion avec sa palette gustative vietnamienne. Voici un repas qu'elle prépare pour marquer le retour de Vincent à Hanoi après un séjour en France :

Vincent a atterri à Hanoi deux jours après moi. Je nous ai préparé une fondue vietnamienne le soir même avec un bouillon clair dans lequel nous avons fait cuire des tranches de poulet, de bœuf, de porc, ainsi que des crevettes et des palourdes. La partie favorite de Vincent était le panier de verdure qui accompagnait les viandes. Sa «mère vietnamienne » m'avait aidée à trouver les rhizomes de nénuphar, les jeunes pousses de bambou, les liserons d'eau, les fleurs de bananier, les fleurs de courge, les ocras, les champignons de paille... et une sorte de mimosa pudique dont le goût et la texture lui plaisaient tout particulièrement. Ce plat était plus goûteux s'il était dégusté en groupe puisque le bouillon s'enrichissait lorsqu'une grande quantité d'ingrédients $y$ cuisaient. Alors même si j'aurais préféré garder Vincent juste pour moi, je l'ai partagé avec nos amis présents à Hanoi. (127-128)

La fondue vietnamienne préparée par Vi pour Vincent et leurs amis, venant de différents horizons, évoque efficacement cette créolisation gustative envisagée par la narratrice, à base d'aliments vietnamiens mélangés à ceux d'autres provenances. Cette longue description, présentant un plat composé de trois sortes de viandes ainsi que deux sortes de fruits de mer avec de nombreux végétaux exotiques sélectionnés avec soin, est destinée à donner une union du plaisir gustatif sans déranger le goût particulier de chaque aliment cuit séparément dans un bouillon clair, contrastant avec la sauce de poisson maternelle qui fut jadis insistante et constante. La complicité et les accords sous-entendus entre les aliments variés de cette fondue ont largement de quoi refléter l'idéal identitaire vers lequel la narratrice est orientée. Bien entendu, cela implique un dépassement des normes gustatives qu'elle a connues avec sa mère dans son cocon maternel, avant de se fusionner dans un nouveau goût transnational. Cette créolisation culinaire est suivie par d'autres descriptions de tables que la narratrice partage avec Vincent et leurs amis d'origines diverses : 
Le dimanche, nous passions trois à quatre heures au brunch gargantuesque de l'hôtel Sofitel, qui offrait une oasis de nourriture introuvable sur le marché local : rosette de Lyon, jambonneau, blanquette de veau, brioches, gravlax, crèmes brûlées, huîtres, cassoulet, coq au vin, baba au rhum, foie gras poêlé, langoustines, Paris-Brest, tarte Tatin, plateau aux mille fromages... Les autres jours de la semaine, nous nous déplacions souvent chez l'un ou l'autre pour faire profiter nos amis de nos trouvailles. Drew, un Australien qui partageait son temps entre 1'Inde et le Vietnam, nous faisait découvrir les épices de la cuisine indienne ; Antoine, Libanais et fin gourmet, savait griller les poissons à la perfection ; Marianne, Brésilienne de Rio, nous préparait plus de cocktails que de nourriture ; Philipp, un Allemand, se montrait toujours ponctuel même dans un pays où le temps était un concept élastique ; Nicholas, notre grand ours polaire, mettait de l'amour dans tout... Autour de la table, nous atteignions souvent le nombre de pays membres du Conseil de sécurité des Nations unies, occupant des professions diverses et possédant des centaines d'histoires à raconter.

La vie véritablement multiculturelle et transnationale que Vi partage avec Vincent et leurs amis, apparait grâce aux plats qu'elle prend au brunch de l'hôtel international, contrastant avec le choix gustatif et olfactif monotone qu'elle mangeait dans l'appartement de sa mère au Québec. L'ouverture vers d'autres cultures qui forme la personne de Vi dans sa nouvelle vie à Hanoi, est également accentuée par l'énumération de ses amis multinationaux qui sont présentés tous avec des pratiques culturelles au-delà de leur particularité culturelle d'origine en créant un ensemble dont les éléments font preuve de l'inter-pénétrabilité tout en préservant leur particularité, tout comme la fondue vietnamienne que Vi leur avait préparée. Ainsi, la quête identitaire de $\mathrm{Vi}$ aboutit dans ce cadre d'ouverture aux autres pratiques culturelles insinuées dans ce choix culinaire symbolique présenté à la fin du récit romanesque.

Pour conclure, les images culinaires sont des moyens efficaces qui permettent d'appréhender la formation identitaire que parcourt la vie migrante de la narratrice Vi. Dans l'écriture de Thúy, la perméabilité des frontières entre l'intérieur et l'extérieur de l'image de soi se concrétise grâce à ces images culinaires symboliques. Le roman vi marque également une évolution au sein de l'écriture de Thúy, qui d'abord a été une «élaboration littéraire du déracinement », comme constaté dans l'analyse de Dusaillant-Fernandez («Du Vietnam au Québec » 75), avant de devenir une élaboration littéraire d'une fusion interculturelle qui s'opère efficacement dans ce roman. Dans cette écriture migrante, Thúy emprunte aux images culinaires un véhicule capable d'évoquer ce qui se trame à l'intérieur du protagoniste passant par une reconnaissance de la vietnamité qui forme l'essentiel identitaire afin d'arriver à une reconnaissance identitaire qui aboutit à une fusion interculturelle. Ainsi, comme nous avons pu le constater, la construction identitaire de Vi est subtilement extériorisée par les diverses images culinaires que l'auteure use finement dans ce roman. 


\section{Ouvrages cités}

Carrière, Marie. «Mémoire du Care, féminisme en mémoire ». Women in French Studies (2015) : 205-217.

Delaney, Brigid. «Why I Can’t Live without Fish Sauce : Author Kim Thúy on the Tastes of Vietnam ». The Guardian. Web. 27 mai 2015.

Dusaillant-Fernandes, Valérie. «Du Vietnam au Québec : fragmentation textuelle et travail de mémoire chez Kim Thúy ». Women in French Studies 20 (2012) : 75-89.

---. « Habiller le vécu de mots et d'images : le projet de Kim Thúy - Entretien avec Kim Thúy ». Voix Plurielles 9.2 (2012) : 164-177.

Gaudette, Jessica. «L'anthropophagie dans les littératures des Amériques ». Rencontres multiculturelles : imprévus et cö̈ncidences. Dir. Patrick Imbert. Ottawa : PU d'Ottawa, $2013: 183-221$.

Harel, Simon. Braconnages identitaires - Un Québec palimpseste, Montréal : VLB, 2006.

Plamondon, Jean-François. «En remontant les rus de l'enfance : interview avec Kim Thúy ». Francofonia 60 (2011) : 149-160.

Robin, Régine. «L'écriture migrante. Est-ce que cela a encore un sens à l'heure d'une littérature-monde en français ?». Trajectoires et dérives de la littérature-monde: poétiques de la relation et du divers dans les espaces francophones. Dir. Cécilia Francis et Robert Viau. $2013:$ 55-71.

Paterson, Janet M. «Identité et altérité : littératures migrantes ou transnationales ? ». Interfaces Brasil/Canadà, Rio Grande 9 (2008) : 87-101.

Sing, Pamela V. « Migrance, sensorium et translocalité chez Ying Chen et Kim Thúy ». International Journal of Francophone Studies 16.3 (2013) : 281-301.

Thúy, Kim. vi, Montréal, Libre Expression, 2016.

--- . Ru, Montréal, Libre Expression, 2009.

--- À toi, Montréal, Libre Expression, 2011.

--- . Mãn, Montréal, Libre Expression, 2013. 